\title{
Studies of nanostructuring in cesium magnesium tungsten phosphate glass
}

\author{
M. Maczka ${ }^{\text {a }}{ }^{*}$, L. Kepinski $^{\text {a }}$, J. Hanuza ${ }^{\text {a,b }}$, S. Kojima ${ }^{\text {c }}$ \\ ${ }^{a}$ Institute of Low Temperature and Structure Research, Polish Academy of Sciences, P.O. Box 1410, \\ 50-950 Wroc.aw 2, Poland \\ ${ }^{\mathrm{b}}$ Department of Bioorganic Chemistry, Faculty of Industry and Economics, Wroc.aw University of \\ Economics, \\ 118/120 Komandorska Street, 53-345 Wroc.aw, Poland \\ c Institute of Materials Science, University of Tsukuba, Tsukuba, Ibaraki 305-8573, Japan
}

\begin{abstract}
$\mathrm{Cs}_{2} \mathrm{OMgOWO} \mathrm{P}_{2} \mathrm{O}_{5}$ glass was examined in a broad temperature range using heat capacity, Raman and Brillouin scattering methods. The main aim was to study the elastic properties of the as-prepared glass, the mechanism of nanostructuring in this material and the in.uence of nanostructuring on its elastic properties. It was shown that this glass exhibits prominent nanocrystallization during thermal treatment, giving $\mathrm{Cs}\left(\mathrm{Mg}_{0.25} \mathrm{~W}_{1.75}\right) \mathrm{O}_{6}$ nanocrystals with the size of $410 \mathrm{~nm}$. The crystallization process was hindered when crystallinity reached about $14 \pm 0.6 \mathrm{wt} \%$. The origin of this behavior was discussed. It was also demonstrated that the elastic properties are largely improved by nanocrystallization.
\end{abstract}

PACS: 63.50.+x; 81.07.Bc; 65.60.+a; 78.35.+c; 62.20.Dc

Keywords: Acoustic properties; Brillouin scattering; Raman scattering; Nanocomposites; Phosphates

* Corresponding author. Fax: +48 713441029.

E-mail address: m.maczka@int.pan.wroc.pl (M. Maczka). 


\section{Introduction}

There is a growing interest in the studies of nanostructures both from theoretical and practical point of view since it is now well known that these materials exhibit many novel and useful properties. Although there are numerous reports on the properties of nanomaterials, much smaller attention has been paid to understand the properties of nanomaterials embedded within an amorphous solid. Such composite materials can be obtained by crystallization of glass. It was shown that depending on the experimental conditions it is often possible to control the crystallization process and obtain transparent nanocrystallized glasses that may have a high potential for applications in photonic devices [1-4]. It was also shown that a solid matrix may significantly change the properties of the nanoparticles [4-6]. On the other hand, existence of nanoparticles has a major effect on the host matrix. For example, it significantly influuences the elastic properties of the material, and the elastic and mechanical properties of glass may be largely improved through nanocrystallization [2,7]. Understanding embedded nanostructures is therefore of great fundamental and technological interest. In particular, it is of great interest to study the elastic properties of nano crystallized glasses. Such studies are relatively scarce and to the best of our knowledge no in situ Brillouin study of the nanocrystallization process was reported so far. This paper is a part of more general study concerning the synthesis, structure, elastic properties and crystallization behavior of the $\mathrm{M}_{2} \mathrm{MgWO}_{2}\left(\mathrm{PO}_{4}\right)_{2}(\mathrm{M}=\mathrm{K}$, $\mathrm{Rb}, \mathrm{Cs}$ ) phosphates that have structures related to famous optical non-linear material $\mathrm{KTiOPO}_{4}$ (KTP). In the previous work IR, Raman, X-ray, DTA and conductivity studies of the $\mathrm{M}_{2} \mathrm{MgWO}_{2}\left(\mathrm{PO}_{4}\right)_{2}(\mathrm{M}=\mathrm{K}, \mathrm{Rb}, \mathrm{Cs})$ glasses were reported [8]. These studies revealed that the $\mathrm{Cs}_{2} \mathrm{MgWO}_{2}\left(\mathrm{PO}_{4}\right)_{2}$ glass (CsMWPO) crystallizes partially when annealed above $\mathrm{Tg}$ giving transparent nanocomposite [8]. In the present work we report temperature-dependent Brillouin, heat capacity, Raman scattering and transmission electron microscopy studies of the nanostructuring process in the CsMWPO glass. We will show that Brillouin spectroscopy can be an efficient tool to monitor changes occurring during this process.

\section{Experimental}

Preparation of the CsMWPO glass was described in our previous work [8]. For Brillouin and Raman measurements, glass samples were cut into $2 \mathrm{~mm}$ thick parallelepipeds, with their two faces optically polished. Polycrystalline $\mathrm{CsMg}_{0.25} \mathrm{~W}_{1.75} \mathrm{O}_{6}$ was synthesized by heating the stoichiometric mixture of $\mathrm{Cs}_{2} \mathrm{CO}_{3}, \mathrm{MgO}$ and $\mathrm{WO}_{3}$ in the platinum crucible for $40 \mathrm{~h}$ at $850 \mathrm{C}$. Density of the as-prepared and annealed glass samples was determined by standard Archimedes technique using distilled water as the working fluid. The accuracy of the measurement was about $\pm 0.01 \mathrm{~g} / \mathrm{cm} 3$. Transmission electron microscopic (TEM) images were recorded with Philips CM20 Super Twin microscope, which at $200 \mathrm{kV}$ provides $0.25 \mathrm{~nm}$ resolution. Specimens for TEM were prepared by dispersing some powder sample in methanol and putting a droplet of the suspension on a copper microscope grid covered with perforated carbon. Heat capacity was measured using a Thermal Analyst 2000 calorimeter (TA Instruments). The samples were of the order of 15 mg mass. The measurements were made for a modulation frequency of $10 \mathrm{mHz}$ and a peak to peak temperature modulation amplitude of $1 \mathrm{~K}$. The sample was heated at $2 \mathrm{~K} / \mathrm{min}$ from 473 to $873 \mathrm{~K}$ and then cooled down to $473 \mathrm{~K}$. The error in heat capacity change due to the glass transition was estimated based on a linear .t of the data in the liquid and glass states. 
The Brillouin scattering spectra were obtained with a $3+3$ pass tandem Sandercock-type Fabry-Perot interferometer. A conventional photon-counting system and a multichannel analyzer were used to detect and average the signal. Both back-scattering and right-angle symmetric scattering geometries of the experiments were employed for room-temperature measurements. For high-temperature measurements, each sample was placed in a homemade furnace and the scattered light from the samples was collected in symmetric 90 scattering geometry. The free spectral range was 30 and $40 \mathrm{GHz}$ for 90 and 180 scattering geometry, respectively. The errors in Brillouin frequencies and bandwidths were estimated from the fit of the Brillouin peaks to the Voigt function. These errors were taken into account in the error analysis of sound velocities and elastic moduli. Raman spectra were measured in back-scattering geometry with a triple-grating spectrometer (Jobin Yvon T64000) and the $532 \mathrm{~nm}$ excitation. The Raman spectra were recorded with a spectral resolution of $2 \mathrm{~cm}^{-1}$.

\section{Results}

\subsection{Heat capacity}

Heat capacity can be described as having two components, a real component Cp' and an imaginary component Cp" [9-12]. The real component is the true heat capacity of the material, which is related to slow molecular motion. The imaginary component of heat capacity was attributed to kinetic events within the sample, or to dissipation processes relating to entropy production [11,12]. MDSC technique gives information on both real and imaginary parts of $\mathrm{Cp}$ and it is therefore a very suitable technique for studies of glass transitions.

Fig. 1 shows the results obtained for the as-prepared glass. It can be noticed that the real part of heat capacity upon heating shows a sigmoid shape stepwise increase over the 755-791 K range, whereas the imaginary part shows asymmetric, positive peak at $774 \pm$ $1 \mathrm{~K}$. The sigmoid shape and the asymmetric, positive peak are the characteristic features of the approach of the unrelaxed state of a glass towards its relaxed state. The glass transition temperature, Tg, taken at maximum of the Cp' peak is $774 \pm 1 \mathrm{~K}$. The magnitude of heat capacity for glass $\mathrm{Cp}^{\mathrm{g}}$ and liquid $\mathrm{Cp}^{\mathrm{l}}$ at the relevant temperatures can be obtained by extrapolation of the supercooled liquid' s and glass' s heat capacities. However, in the case of the CsMWPO glass this procedure is not possible for the liquid state due to the unusual shape of Cp' curve. Anyway, the value of heat capacity change at the phase transition, $\Delta \mathrm{Cp}$, was roughly estimated as the difference between maximal value of Cp' at $774 \mathrm{~K}$ and the value of Cp' at the onset of the glass transition, i.e. at approximately $730 \mathrm{~K}$. This value is $0.26 \pm 0.02 \mathrm{~J} / \mathrm{gK}(180.9 \pm 13.9 \mathrm{~J} / \mathrm{mol} \mathrm{K})$. Apart from the above discussed features typical for the glass transition, the heat capacity study revealed an additional anomaly, i.e. the Cp' showed a decrease in the temperature range 791-830 K and the Cp” showed the presence of a weak, negative peak near $801 \pm 1 \mathrm{~K}$. In order to obtain some additional information about the properties of this glass, we also performed heat capacity studies of the glass annealed for about $5 \mathrm{~h}$ at $873 \mathrm{~K}$ temperature (see Fig. 1). It is clearly seen from Fig. 1 that the annealed sample does not show the presence of any additional anomaly except of that connected with the glass transition. The value of heat capacity change at the phase transition, $\Delta \mathrm{Cp}$, is much smaller than that observed for the as-prepared sample, i.e. it is only about $0.11 \pm 0.01 \mathrm{~J} / \mathrm{gK}(76.5 \pm 6.9 \mathrm{~J} / \mathrm{mol} \mathrm{K})$. 


\subsection{TEM}

TEM images of the as-prepared sample (not shown) confirmed that it is amorphous with no indications of any long range order. Accordingly, XRD pattern presented in Ref. [8] contained only very broad maximum typical for amorphous materials. Fig. 2 shows high-resolution TEM image of the sample annealed at $873 \mathrm{~K}$ for $5 \mathrm{~h}$. It is seen that the sample is mostly amorphous, but there are also small areas $(4-10 \mathrm{~nm})$ with long range order. Distances between lattice fringes in these ordered areas 0.6 and $0.31 \mathrm{~nm}$ correspond to (111) and (311) interplanar spacings in cubic $\mathrm{CsMg}_{0.25} \mathrm{~W}_{1.75} \mathrm{O}_{6}$ (PDF 00-055-0943). This result is consistent with XRD pattern, where broad reflections at positions expected for $\mathrm{CsMg}_{0.25} \mathrm{~W}_{1.75} \mathrm{O}_{6}$ were observed [8]. It is worth noting that the annealed sample has a very high transparency comparable to the as-prepared glass. According to the scattering theory, the coefficient of scattering is governed by the size of crystals dispersed in amorphous matrix, and the refractive index difference between crystal and matrix. Therefore, much smaller crystals and smaller refractive index difference provide lower scattering. The high transparency of the annealed CsMWPO sample is achieved by the very small size of the precipitated nanocrystals, similar as for the most known transparent glass ceramics.

\subsection{Brillouin}

The unpolarized Brillouin spectra of the as-prepared glass at a few di.erent temperatures are presented in Fig. 3. These spectra show a strong elastic peak due to the scattering from LA modes at about $9 \mathrm{GHz}$ and a second, very weak peak at about $5 \mathrm{GHz}$ due to light scattered from TA modes. The frequency shifts and bandwidths of these peaks were evaluated by fitting the measured spectra to the convolution of the Gaussian instrumental function with a theoretical spectral line shape (Lorenzian). However, because of very weak intensity of the TA modes, their frequencies were evaluated only at room temperature. The spectra presented in Fig. 3 were measured in symmetric 90 scattering geometry [13]. The advantage of this scattering geometry is that it allows sound velocities to be measured without knowing the refractive index of the sample [13]. Velocities were hence directly determined from the Brillouin shifts $v_{B}$ from the relation $V=v_{90} \lambda / 2 \sin 45$, where $\lambda$ is the wavelength of the laser light. In an amorphous solid, elastic strain can be described by two independent elastic constants, $\mathrm{C}_{11}$ and $\mathrm{C}_{44}$. The Cauchy relation $2 \mathrm{C}_{44}=\mathrm{C}_{11}-\mathrm{C}_{12}$ allows one to determine $\mathrm{C}_{12}$. From the values of longitudinal and transverse sound velocities extracted from Brillouin peaks, the constants characterizing the elastic and mechanical properties of a glass can be calculated, i.e. the longitudinal modulus $\mathrm{L}=\mathrm{C}_{11}=\rho \mathrm{V}^{2}$, shear modulus $\mathrm{G}=\mathrm{C}_{44}$ $=\rho V^{2}$, bulk modulus $\mathrm{K}=\mathrm{L}-(4 / 3) \mathrm{G}$, Young's modulus $\mathrm{E}=\mathrm{G}(3 \mathrm{~L}-4 \mathrm{G}) /(\mathrm{L}-\mathrm{G})$ and Poisson's ratio $\sigma=(\mathrm{L}-2 \mathrm{G}) / 2(\mathrm{~L}-\mathrm{G})$, where $\mathrm{V}_{\mathrm{L}}, \mathrm{V}_{\mathrm{T}}$ and $\rho$ denote longitudinal sound velocity, transverse sound velocity and density, respectively. Because the TEM studies revealed that the crystalline sizes of nanocrystals in the annealed glass are around 4-10 nm, this glass was treated as isotropic material in this study. The calculated elastic constants for the as-prepared and nanocrystallized glasses, using the densities determined by us, are summarized in Table 1. It is clear that the values of sound velocities, bulk, shear and Young's moduli in the nanocrystallized glass are larger than those in the as-prepared glass. For instance, the bulk modulus of the nanocrystallized glass is $33.7 \pm 0.7 \mathrm{GPa}$, but the as-prepared glass has a value of $27.5 \pm 0.5 \mathrm{GPa}$, giving $22.5 \%$ increase due to the 
nanocrystallization.

Fig. 4 shows the temperature dependence of longitudinal acoustic mode for the as-prepared sample. It can be noticed that in the glass state temperature dependence is weak and linear. The linear .t of $\mathrm{V}_{\mathrm{L}}\left(\mathrm{V}_{\mathrm{L}}=\mathrm{A}+\mathrm{BT}\right)$ yields $\mathrm{A}=3390.3 \pm 4.9$ and $\mathrm{B}=\_0.248 \pm$ 0.016 . When temperature reaches approximately $\mathrm{Tg}-100 \mathrm{~K}$, a change in the regime of the $\mathrm{V}_{\mathrm{L}}$ dependence with temperature is observed, i.e. $\mathrm{V}_{\mathrm{L}}$ shows non-linear increase from $\mathrm{Tg}$ -100 to Tg. This increase, estimated as a difference between the experimental value of $\mathrm{V}_{\mathrm{L}}$ at $\mathrm{Tg}$ and the value of $\mathrm{V}_{\mathrm{L}}$ at $\mathrm{Tg}$ calculated from the given above linear fit, is about $2.7 \%$. At around $774 \mathrm{~K}$ the slope of $\mathrm{V}_{\mathrm{L}}$ vs temperature changes sharply. Such behavior is generally observed in glass transitions and indicates that the studied material above $774 \mathrm{~K}$ is in the supercooled liquid state. The linear fit of $\mathrm{V}_{\mathrm{L}}$ in the 774-808 $\mathrm{K}$ temperature range yields $\mathrm{A}=$ $5948.9 \pm 140$ and $\mathrm{B}=-3.45 \pm 0.18$. At higher temperatures a significant increase in the $\mathrm{V}_{\mathrm{L}}$ is observed from about $3130 \mathrm{~m} / \mathrm{s}$ at $808 \mathrm{~K}$ to about $3340 \mathrm{~m} / \mathrm{s}$ at $838 \mathrm{~K}$. Above $838 \mathrm{~K}$ the $\mathrm{V}_{\mathrm{L}}$ starts to decrease again but the temperature dependence of $\mathrm{V}_{\mathrm{L}}$ is non-linear. The slope of $\mathrm{V}_{\mathrm{L}}$ vs temperature above $838 \mathrm{~K}$ is steeper than in the $774-808 \mathrm{~K}$ temperature range. When temperature reaches about $960 \mathrm{~K}$, the sample becomes opaque and the Brillouin signal vanishes.

Fig. 5 also shows that the bandwidth of the LA mode increases very slowly in the 300-790 K range, indicating long relaxation time. The bandwidth shows a moderate increase in the 790-890 K range and a fast increase above $890 \mathrm{~K}$ temperature. This result indicates fast decrease of the relaxation time above $890 \mathrm{~K}$. Shorter relaxation time also leads to the asymmetric shape of the LA band at high temperatures.

Fig. 4 also shows the temperature dependence of the Brillouin shift for the nanocrystallized sample (heat treated at $873 \mathrm{~K}$ for $5 \mathrm{~h}$ ). It can be noticed that for this sample no anomalous frequency increase is observed in the supercooled liquid state. The glass transition is observed clearly through change in the slope of $\mathrm{V}_{\mathrm{L}}$ vs temperature, and the temperature dependence of velocity $\mathrm{V}_{\mathrm{L}}$ above $840 \mathrm{~K}$ is nearly the same for the nanocrystallized and as-prepared samples.

It is also worth noting that the sample used in the Brillouin studies and heated up to $960 \mathrm{~K}$ temperature preserved its form. This behavior is very di.erent from the behavior of the $\mathrm{K}_{2} \mathrm{MgWO}_{2}\left(\mathrm{PO}_{4}\right)_{2}\left(\mathrm{Rb}_{2} \mathrm{MgWO}_{2}\left(\mathrm{PO}_{4}\right)_{2}\right)$ glass, for which significant softening and deformation were observed already at temperatures higher than about $\mathrm{Tg}+50 \mathrm{~K}(\mathrm{Tg}+30$ $\mathrm{K})$. This comparison shows that the viscosity of the CsMWPO sample remains very high well above Tg. High viscosity above Tg can be attributed to the presence of nanocrystallites since it is well known that the viscosity of a composite material composed of nanocrystallites embedded in amorphous matrix increases with the nanocrystallite volume fraction $[14,15]$.

\subsection{Raman spectra}

Temperature-dependent Raman spectra of the CsMWPO glass are shown in Figs. 6 and 7. The assignment of the room-temperature bands was already discussed in our previous paper [8] and here we only remind that the most intense bands at 930 and $373+$ $260 \mathrm{~cm}^{-1}$ correspond to stretching and bending vibrations of the $\mathrm{WO}_{6}$ units, whereas the 10641203 and $743 \mathrm{~cm}^{-1}$ bands indicate the presence of some pyrophosphate and metaphosphate groups. The recorded spectra also show the presence of a broad Boson band 
with maximum at about $33 \mathrm{~cm}^{-1}$.

When temperature is raised, the Raman spectra do not show any significant changes up to $790 \mathrm{~K}$. In contrast, when the sample is heat treated at $830 \mathrm{~K}$ significant changes occur in the spectra. First of all, new Raman bands appear near 725 and $200 \mathrm{~cm}^{-1}$. Moreover, it can be noticed that some changes occur in the low-frequency region of the Raman spectra, i.e. scattering intensity below $30 \mathrm{~cm}^{-1}$ increases significantly, a new band appears at $55 \mathrm{~cm}^{-1}$ in the $\mathrm{VV}$ polarization and the spectra recorded in the $\mathrm{VH}$ polarization seems to be composed of two components. It is worth noting that the intensity of the $930 \mathrm{~cm}^{-1}$ band is weaker in the annealed glass by only about $22 \pm 1 \%$ in comparison with the as-prepared one. Since this band is informative on the concentration of $\mathrm{WO}_{3}$ oxide in the amorphous matrix, it is clear that the remaining amorphous matrix has still a large concentration of $\mathrm{WO}_{3}$. In order to isolate the contribution of the nanocrystals, it is necessary to subtract the Raman scattering contribution of the glass matrix. Since the crystalline nucleation reduced the $\mathrm{WO}_{3}$ content in the glass matrix, spectra of the nanocrystals were obtained by subtracting a proper fraction $(0.78 \pm 0.01)$ of the VV $(\mathrm{VH})$ spectrum obtained for the same sample at $790 \mathrm{~K}$ temperature. Fig. 8 shows the results of this procedure. As presented in Fig. 8 both in VV and VH polarization the most intense bands are observed at 55, 200, 725 and $900 \mathrm{~cm}^{-1}$.

\section{Discussion}

\subsection{Properties of the as-prepared glass and study of the glass transition}

The comparison between the CsMWPO and the previously studied $\mathrm{K}_{2} \mathrm{MgWO}_{2}\left(\mathrm{PO}_{4}\right)_{2}(\mathrm{KMWPO})$ and $\mathrm{Rb} 2 \mathrm{Mg}-\mathrm{WO}_{2}\left(\mathrm{PO}_{4}\right)_{2}$ (RbMWPO) glasses shows that $\mathrm{Tg}$ is lowered by $21-23 \mathrm{~K}$ when $\mathrm{K}+$ or $\mathrm{Rb}+$ ions are replaced by Cs+ ions [8]. This result indicates a decrease in network connectivity in the CsMWPO. The relative change of heat capacity at the glass transition is large (the $\mathrm{C}_{\mathrm{l}}=\mathrm{C}_{\mathrm{gp}}$ ratio is $1.38 \pm 0.03$ ). A number of studies showed that such a large increase of $\mathrm{C} 0 \mathrm{p}$ is a characteristic feature of a fragile liquid [9,16-18], indicating that the CsMWPO phosphate is a relatively fragile liquid. Recently, a correlation between the dynamic and thermodynamic fragilities has been re-examined for a number of glass-formers [18]. It has been shown that inorganic glass-forming liquids exhibit the behavior originally described by Angell [16] that the dynamic fragility index $m$ increases as the ratio $\mathrm{C}_{\mathrm{p}}=\mathrm{C}_{\mathrm{gp}}$ increases. According to Huang' $\mathrm{s}$ correlation between $\mathrm{m}$ and $\mathrm{C}_{\mathrm{p}}=\mathrm{C}_{\mathrm{gp}}$ [16] the fragility index $\mathrm{m}$ should be about $49 \pm 2$ for the CsMWPO glass. Although heat capacity study cannot provide very accurate value of fragility index, which should in principle be obtained from the temperature dependence of viscosity, value estimated by us indicates that this phosphate belongs to the group of materials exhibiting intermediate fragility.

Brillouin study revealed that Poisson's ratio of the studied glass is $0.274 \pm 0.007$. Previous studies on the relation Fig. 7. Low-frequency VH Raman spectra at di.erent temperatures corrected for their frequency and temperature dependence using Eq. (1). The spectra were vertically shifted for clarity sake. of Poisson's ratio with the glass structure showed that $r$ is greater than 0.25 when the ions themselves are deformed under stress in addition to the network distortion [19-21]. Moreover, the $L / G$ (or $G / C_{12}$ ) ratio is an indicator of the character of the force .eld: if atoms interact through a central potential, $\mathrm{G} / \mathrm{C}_{12}=1(\mathrm{~L} / \mathrm{G}=3)$, if through non-central potential, $\mathrm{G} / \mathrm{C}_{12}<1(\mathrm{~L} / \mathrm{G}>3)[22,23]$. The 
$\mathrm{G} / \mathrm{C}_{12}$ value is $0.82 \pm 0.04$, indicating non-central forces. These values are between that of pure $\mathrm{P}_{2} \mathrm{O}_{5}$ glass (0.7) and $\mathrm{Fe}_{2} \mathrm{O}_{3}-\mathrm{P}_{2} \mathrm{O}_{5}$ glass (values close to 1) [22].

The temperature-dependent Brillouin study shows that the rigidity of the glass network markedly decreases with increasing temperature, as evidenced through a decrease of longitudinal sound velocity with increasing temperature. The rate of decrease is significantly higher above Tg. Such a behavior is generally observed in glass transitions and can be attributed to the fact that the temperature dependence of viscosity in a supercooled liquid state is higher than in a glassy state. It is worth noting, however, that the longitudinal sound velocity also exhibits a non-linear increase in the region $674774 \mathrm{~K}$. This behavior is very similar to the behavior reported for $\mathrm{ZnO}-\mathrm{PbO}-\mathrm{P}_{2} \mathrm{O}_{5}$ glass [24] which was attributed to the reorganization of glass due to annealing process which removed residual stresses in the asmelted samples [24]. Our result indicates, therefore, that the as-prepared CsMWPO glass is characterized by large residual stresses having large relaxation times. The relative large change in VL near Tg demonstrates relatively loose random packed microstructure of the as-prepared CsMWPO glass.

It is well known that a characteristic feature of a glass is the appearance of the so-called Boson peak corresponding to an excess of low-frequency vibrational states in respect to the predictions of the Debye theory that can be observed in the Raman spectra below $120 \mathrm{~cm}^{-1}$ [25 28]. The spectra of the CsMWPO glass presented in Fig. 7 are shown in the reduced form:

$$
I^{\mathrm{red}}(\omega)=\frac{I^{\exp }(\omega)}{\omega[n(\omega, T)+1]} \propto \frac{g(\omega) C(\omega)}{\omega^{2}}
$$

where $I^{\text {exp }}(\omega), C(\omega)$, g $(\omega)$ and $n(\omega, T)$ denote the experimentally measured scattered Raman intensity, the photon-phonon or Raman coupling coefficient, vibrational density of states and the Bose-Einstein occupation number, respectively. The maximum of the observed Boson peak is relatively low, near $33 \mathrm{~cm}^{-1}$. Very similar value, $30 \mathrm{~cm}^{-1}$, was previously found for $\mathrm{CsPO}_{3}$ [29]. Change of the Boson peak intensity and frequency was very often related to changes in some glass properties. For instance, it has been claimed that the frequency of the Boson peak can be related to the length scale at which the molecular structure becomes important [30,31]. Recently, the validity of empirical correlations between a spectral characteristic of the Boson peak and a specific property of the glass was questioned since it was shown that the maximum frequency of the Boson peak is not directly related to the maximum distribution of the excess of states [28]. On the contrary, it is sensitive to the type of the tails of the low-frequency edge of such a distribution. It has been shown that the change of the excess distribution tails from convex (semicircular type) to concave (Gaussian) will result in reducing of the Boson peak asymmetry and shifting the frequency of its maximum to higher energy [28]. Another problem is that the spectral characteristic of the Boson peak is influenced by the contribution of the Debye density of states $\left(g^{\mathrm{D}}(\omega)\right)$ [28]. It was also shown that the shift of the excess of states to lower frequencies will result in artificial increase of the reduced Boson peak intensity as well as the appearance of the wing at frequencies close to zero, reminiscent of the quasi-elastic scattering in the low frequency Raman spectra [28]. Having these facts in mind, it is clear 
that the true excess density of states, i.e. $g(\omega)^{\text {exc }}=g(\omega)-g^{D}(\omega)$ should be analyzed in order to obtain some information about glass properties. The $g^{\mathrm{D}}(\omega)$ can be easily calculated from the formula: $g^{D}=3 \omega^{2} / \omega^{D}$, where the Debye frequency can be estimated from the Brillouin data. Unfortunately, Raman intensity is weighted by the $\mathrm{C}(\omega)$ coupling coe.cient and in order to estimate the absolute value of $g(\omega)$ from Raman scattering a proper normalization factor is needed which can be derived from the low-temperature heat capacity studies [32]. Since such data are not available for the studied glass, we cannot estimate the $g(\omega)^{\text {exc }}$ from our Raman measurements. We may, however, conclude that the observed increase of the Boson peak intensity with increasing temperature can be explained by the shift of the $g(\omega)^{\text {exc }}$ towards lower frequencies and increased contribution of the $\mathrm{g}^{\mathrm{D}}(\omega)$ term. The shift of the $\mathrm{g}(\omega)^{\mathrm{exc}}$ towards lower frequencies may also explain the observed intensity increase on the low-frequency side of the Boson peak, which resembles quasi-elastic scattering.

\subsection{Nanocrystallization}

As mentioned above, the real component of heat capacity decreases above $791 \mathrm{~K}$ and the imaginary component of heat capacity, C” p , exhibits a negative peak near $801 \mathrm{~K}$. It is well known that C" p arises from the out-of-phase response of the sample [9,12]. This response varies in sign for different transitions. Melts and glass-liquid transitions are typically endothermic events and are observed in C” p plots as positive peaks [9,12]. On the other hand, the exothermic cold crystallization exhibits a negative peak [12]. Because TEM and X-ray studies clearly showed that the sample partially crystallizes when annealed above $800 \mathrm{~K}$, the additional anomaly observed by us in C” p must be attributed to a partial nanocrystallization of the sample. Since heat capacity of a crystal is smaller than that of a liquid, the decrease of C'p above $791 \mathrm{~K}$ is observed due to the onset of the crystallization. The absence of this additional peak in the heat capacity plot obtained for the sample annealed at $873 \mathrm{~K}$ for $5 \mathrm{~h}$ indicates that the crystallization has been completed during the annealing. As mentioned above, the value of heat capacity change at the phase transition, $\Delta \mathrm{Cp}$, is much smaller for the nanocrystallized sample (about $0.11 \pm 0.01 \mathrm{~J} / \mathrm{g} \mathrm{K}$ ) than for the as-prepared sample (about $0.26 \pm 0.02 \mathrm{~J} / \mathrm{g} \mathrm{K}$ ). Such a large change could suggest that most of the sample is now in crystalline state. However, it is clear from the Raman, Brillouin and TEM studies that most of the sample is still in amorphous phase. Indeed, the comparison of the Raman intensity of the $930 \mathrm{~cm}^{-1}$ band for the as-prepared and annealed sample shows that the concentration of the $\mathrm{WO}_{3}$ oxide changed from $25 \%$ in the as-prepared glass to about $19.5 \pm 0.25 \%$ in the amorphous matrix of the annealed sample. Describing the phase separation of the glass into the amorphous matrix and $\mathrm{Cs}\left(\mathrm{Mg}_{0.25} \mathrm{~W}_{1.75}\right) \mathrm{O}_{6}$ nanocrystallites by the following reaction:

$$
\mathrm{Cs}_{2} \mathrm{O}-\mathrm{MgO}-\mathrm{WO}_{3}-\mathrm{P}_{2} \mathrm{O}_{5} \rightarrow x \mathrm{Cs}\left(\mathrm{Mg}_{0.25} \mathrm{~W}_{1.75}\right) \mathrm{O}_{6}+(1-0.5 x) \mathrm{Cs}_{2} \mathrm{O}-(1-0.25 x) \mathrm{MgO}-(1-1.75 x) \mathrm{WO}_{3}-\mathrm{P}_{2} \mathrm{O}_{5}
$$

one fineds $\mathrm{x}=0.174 \pm 0.007$, which means that only about $30.5 \pm 1.3 \%$ of $\mathrm{WO}_{3}$ was used for crystallization of the pyrochlore phase and that the nanocrystallized sample contains about $14 \pm 0.6 \mathrm{wt} \%$ of $\mathrm{Cs}\left(\mathrm{Mg}_{0.25} \mathrm{~W}_{1.75}\right) \mathrm{O}_{6}$ pyrochlore. The large change in $\Delta \mathrm{C}$ 'p has to be, therefore, attributed to both partial crystallization of the sample as well as change 
in chemical composition of the remaining amorphous matrix. It is also worth noting that the glass transition region is larger for the annealed glass, due to large inhomogeneities of the sample. The glass transition of the annealed sample is shifted to higher temperatures by only $2 \mathrm{~K}$ indicating that thermal stability of the remaining amorphous matrix is very similar to that of the initial amorphous phase. The development of crystalline particles in the bulk amorphous matrix leads to increase of the rigidity of the studied material, as evidenced through the increase of longitudinal sound velocity of the nanocrystallized sample. Brillouin study shows that the precipitation of the nanocrystallites starts at about $808 \mathrm{~K}$. It was previously found that the existence of nanoparticles dispersed in amorphous matrix leads to an increase of the sound velocity and elastic moduli [2,7]. It is, however, di.cult to predict the magnitude of this increase because elastic properties of a partially crystallized sample depend on many factors such as elastic properties of the nanocrystals and the matrix, volume fraction of nanocrystals, their size and shape [2,7]. Moreover, it is known that contact e.ects between nanocrystals and the matrix or a non-homogenous distribution of the nanocrystals may also lead to some changes in elastic behavior. In our case, the increase of sound velocity is observed in the $808-838 \mathrm{~K}$ temperature range indicating that the volume fraction of the pyrochlore nanoparticles increases continuously in this range. The whole of transformations occurring in the glass matrix during the Brillouin experiment can be considered as complete at about $838 \mathrm{~K}$, i.e. after about $2 \mathrm{~h}$, and the sound velocity decreases again due to decrease in viscosity of the composite material.

It is worth noting that the temperature dependence of sound velocity in the as-prepared and the annealed samples is nearly the same above $840 \mathrm{~K}$ temperature. This result indicates that the crystallization process is completed during the Brillouin experiment of the as-prepared sample or after a prolonged annealing at $873 \mathrm{~K}$, and that the main a-relaxation is clearly observed for the partially crystallized samples. It is, therefore, clear that the behavior of this composite is markedly determined by the amorphous matrix, and the crystalline particles do not form a rigid continuous network but are embedded in this amorphous matrix. This conclusion is consistent with our estimation that the nanocrystallized sample contains only $14 \pm 0.6 \mathrm{wt} \%$ of nanoparticles. A question then arises which mechanism leads to the formation of nanocrystals and why the crystallization is hindered when only about $30.5 \pm 1.3 \%$ of $\mathrm{WO}_{3}$ is used. One of the possible mechanism is known to be the extremely high nucleation rate and small growth rate and another is the phase separation on extremely .ne scale in supercooled liquid [1,33-37]. Actually, the nanoscale separation in supercooled liquid is a common phenomenon in many materials exhibiting nanocrystallization. This mechanism was observed, for example, in $\mathrm{K}_{2} \mathrm{O}-\mathrm{Pb}_{2} \mathrm{O}_{5}-\mathrm{SiO}_{2}$ and $\mathrm{SiO}_{2}-\mathrm{Al}_{2} \mathrm{O}_{3}-\mathrm{Pb}_{2} \mathrm{O}-\mathrm{B}_{2} \mathrm{O}_{3}-\mathrm{Na}_{2} \mathrm{O}$ glasses as well as in many metallic glasses $[1,36,37]$. When phase separation occurs on large scale prior to crystallization, appearance of additional Brillouin peaks should be observed. This behavior was indeed observed, for instance, for triphenyl phosphate for which appearance of the second amorphous phase was evidenced by appearance of a second Brillouin peak corresponding to longitudinal phonon that grew in intensity with annealing time [38]. Because during our experiment, narrow Brillouin peak is observed, it is clear that only one amorphous phase is present, i.e. no separation into two amorphous phases occurs on large scale in this material during heat treatment. A possible scenario for nucleation of the pyrochlore phase is, therefore, the formation of small amount of tungsten-rich clusters due to local exchange of 
atoms. When the composition of these clusters becomes close to the $\mathrm{CsMg}_{0.25} \mathrm{~W}_{1.75} \mathrm{O}_{6}$ pyrochlore, the amorphous to crystalline phase transformation is triggered. As disscussed elsewhere, when crystallization of a phase with different composition than the amorphous matrix occurs, very fine nanocrystalline structures can be obtained $[39,40]$. This is due to the fact that this process is controlled by long-range diffusion of the components. This discusion becomes important only when the viscosity of the network is sufficiently low. As a result, the nanoparticles of the $\mathrm{CsMg}_{0.25} \mathrm{~W}_{1.75} \mathrm{O}_{6}$ pyrochlore start to grow when the temperature is higher than $\mathrm{Tg}+17$ (higher than $791 \mathrm{~K}$ ). The long-range diffusion is also responsible for the fact that the growth rate is slowing down with increasing size of a crystal and change in the chemical composition of the remaining amorphous matrix. When the diffusion fields overlap, the further growth of the crystallites is hindered, regardless of the heat treatment time. This mechanism explains why the nanoparticles in our glass are very small in spite of long annealing time and why only about $30.5 \pm 1.3 \%$ of $\mathrm{WO}_{3}$ is used for precipitation of the pyrochlore. As mentioned above, the most intense Raman bands of the nanocrystalline phase are observed near 55, 200, 725 and $900 \mathrm{~cm}^{-1}$. The spectrum, recorded for the synthesized $\mathrm{CsMg}_{0.25} \mathrm{~W}_{1.75} \mathrm{O}_{6}$ pyrochlore, exhibits similar bands (see Fig. 8), confirming that the nanocrystals have a pyrochlore structure. There are, however, some important differences between the spectra of the nanocrystallized material and the synthesized pyrochlore. Firstly, the very broad band, which can be attributed to the stretching vibrations of the $\mathrm{W}-\mathrm{O}-\mathrm{W}$ bridges, shifts from about $700 \mathrm{~cm}^{-1}$ for the $\mathrm{CsMg}_{0.25} \mathrm{~W}_{1.75} \mathrm{O}_{6}$ pyrochlore to $725 \mathrm{~cm}^{-1}$ for the nanocrystallized material. The origin of this shift is not clear but we suppose that it may be due to some changes in the $\mathrm{W}$-O bond lengths and $\mathrm{O}-\mathrm{W}-\mathrm{O}$ bond angles with decreasing size of the crystallites. Secondly, signi.cant change in the bandwidth of the $55 \mathrm{~cm}^{-1}$ band is observed from $16.0 \mathrm{~cm}^{-1}$ for the $\mathrm{CsMg}_{0.25} \mathrm{~W}_{1.75} \mathrm{O}_{6}$ pyrochlore to $35.9 \mathrm{~cm}^{-1}$ for the nanocrystals. This large increase in the bandwidth is consistent with small size of the nanocrystals. The comparison of the obtained spectra also shows that all the additional bands of the nanocrystalline samples have counterparts in the spectra of the synthesized pyrochlore crystallites, i.e. we do not observe any Raman bands which could be assigned to confined acoustic phonon vibrational modes. Such low-frequency modes were observed in many composites composed of nanocrystals embedded in an amorphous matrix [41-43]. The absence of these modes in our spectra, in spite of the small size of the nanocrystals, may indicate that either intensity of these modes is weak, and they are obscured by the stronger band near $55 \mathrm{~cm}^{-1}$, or they appear at very low frequency, below the limit of our experimental setup.

\section{Conclusions}

The performed study revealed that the CsMWPO glass is not stable and near $791 \mathrm{~K}$ pyrochlore type $\mathrm{CsMg}_{0.25} \mathrm{~W}_{1.75} \mathrm{O}_{6}$ nanocrystals start to precipitate. A highly transparent glass ceramics can be easily obtained by annealing the samples in the $800873 \mathrm{~K}$ range. The crystallization process is complete at about $840 \mathrm{~K}$ after $2 \mathrm{~h}$ of annealing. The crystallite size and percent crystallinity of the sample annealed at $873 \mathrm{~K}$ for $5 \mathrm{~h}$ are $410 \mathrm{~nm}$ and about $14 \pm 0.6 \mathrm{wt} \%$, respectively. The elastic properties of this glass ceramics are typical of a material in which nanoparticles do not form a rigid continuous network but are embedded in an amorphous matrix. Indeed, the longitudinal sound velocity still exhibits the main a-relaxation characteristic of amorphous materials. However, existence of 
nanoparticles leads to significant increase in elastic moduli. This increase is up to $22.5 \%$ in the case of bulk modulus. The Brillouin studies give strong evidence that the primary crystallization is not preceded by large scale phase separation in the supercooled liquid region. The separation occurs only on small scale followed by fast crystallization of the tungsten-rich clusters.

\section{Acknowledgments}

This work was supported by the Polish Committee for Scientific Research, Grant No. 1 PO3B 078 29. Dr M. Maczka acknowledges Tsukuba University for support of his stay in Japan and Dr A. Hushur, University of Tsukuba, for his help in performing the Brillouin measurements.

\section{References}

[1] A. Aronne, V.N. Sigaev, B. Champagnon, E. Fanelli, V. Califano, L.Z. Usmanova, P. Pernice, J. Non-Cryst. Solids 351 (2005) 3610.

[2] F. Torres, K. Narita, Y. Benino, T. Fujiwara, T. Komatsu, J. Appl. Phys. 94 (2003) 5265.

[3] I. Enomoto, Y. Benino, T. Fujiwara, T. Komatsu, J. Solid State Chem. 179 (2006) 1821.

[4] K.E. Lipinska-Kalita, B. Chen, M.B. Kruger, Y. Ohki, J. Murowchick, E.P. Gogol, Phys. Rev. B 68 (2003) 35209.

[5] R. Goswami, K. Chattopadhyay, Appl. Phys. Lett. 69 (1996) 910.

[6] Y.M. Yang, X.L. Wu, L.W. Yang, G.S. Huang, T. Qiu, Y. Shi, J. Appl. Phys. 99 (2006) 14301.

[7] F. Torres, Y. Benino, T. Fujiwara, T. Komatsu, Mater. Res. Bull. 39 (2004) 1431.

[8] M. Maczka, J. Hanuza, A. Hushur, S. Kojima, J. Non-Cryst. Solids 352 (2006) 5586.

[9] O. Bustin, M. Descamps, J. Chem. Phys. 110 (1999) 10982.

[10] E. Tombari, S. Presto, G. Salvetti, G.P. Johari, J. Chem. Phys. 117 (2002) 8436.

[11] R. Haase, Thermodynamics of Irreversible Processes, Dover Publications, New York, 1990.

[12] S.R. Aubuchon, P.S. Gill, J. Therm. Anal. 49 (1997) 1039.

[13] C.H. Whit.eld, E.M. Brody, W.A. Bassett, Rev. Sci. Instrum. 47 (1976) 942.

[14] V. Yong, H.T. Hahn, Nanotechnology 15 (2004) 1338.

[15] A.R. Boccaccini, Mater. Lett. 34 (1998) 285.

[16] C.A. Angell, Strong and fragile liquids, in: K. Ngai, G.B. Wright (Eds.), Relaxation in Complex Systems, Naval Research Laboratory, Washington, DC, 1985.

[17] K. Ito, C.T. Moynihan, C.A. Angell, Nature 398 (1999) 492.

[18] D. Huang, G.B. McKenna, J. Chem. Phys. 114 (2001) 5621.

[19] T. Smyth, J. Am. Ceram. Soc. 42 (1959) 277.

[20] B. Bridge, N.D. Patel, D.N. Waters, Phys. Status Solidi A 77 (1983) 655.

[21] C.C. Chen, Y.J. Wu, L.G. Hwa, Mater. Chem. Phys. 65 (2000) 306.

[22] K.H. Chang, T.H. Lee, L.G. Hwa, Chin. J. Phys. 41 (2003) 414.

[23] M. Born, K. Huang, Dynamical Theory of Crystal Lattices, University Press, Oxford, 1966.

[24] G. Le Saou t, Y. Vaills, Y. Luspin, Solid State Commun. 123 (2002) 49.

[25] W. Schirmacher, G. Diezemann, C. Ganter, Phys. Rev. Lett. 81 (1998) 136.

[26] S.N. Taraskin, Y.L. Loh, G. Natarajan, S.R. Elliot, Phys. Rev. Lett. 86 (2001) 1255. 
[27] A.I. Chumakov, I. Sergueev, U. Van Bu “ rck, W. Schirmacher, T. Asthalter, R. Ru “ .er, O. Leupold, W. Petry, Phys. Rev. Lett. 92 (2004) 245508.

[28] S.N. Yannopoulos, K.S. Andrikopoulos, G. Ruocco, J. Non-Cryst. Solids 352 (2006) 4541.

[29] P.J. Miller, J. Chem. Phys. 71 (1979) 997.

[30] S.R. Elliot, in: Physics of Amorphous Materials, Longman Science and Technical, 1990, p. 139.

[31] C. Levelut, N. Gaimes, F. Terki, G. Cohen-Solal, J. Pelous, R. Vacher, Phys. Rev. B 51 (1995) 8606.

[32] A.P. Sokolov, A. Kisliuk, D. Quitmann, Phys. Rev. B 48 (1993) 7692.

[33] T. Kulik, J. Non-Cryst. Solids 287 (2001) 145.

[34] Y.T. Wang, Z.Y. Pang, R.J. Wang, D.Q. Zhao, M.X. Pan, B.S. Han, W.L. Wang, W.H. Wang, J. Non-Cryst. Solids 352 (2006) 444.

[35] U. Ko “ $\quad$ stner, R. Janlewing, Mater. Sci. Eng. A 375-377 (2004) 223.

[36] S. Morimoto, J. Non-Cryst. Solids 352 (2006) 756.

[37] W.L. Johnson, Mater. Res. Bull. 24 (1999) 42.

[38] J. Senker, E. Ro “* ssler, Chem. Geol. 174 (2001) 143.

[39] A.L. Greer, Mater. Sci. Eng. A 179\&180 (1994) 41.

[40] D. Crespo, T. Pradell, N. Clavaguera, M.T. Clavaguera-Mora, Mater. Sci. Eng. A 238 (1997) 160.

[41] A. Tanaka, S. Onari, T. Arai, Phys. Rev. B 47 (1993) 1237.

[42] A. Roy, A.K. Sood, Solid State Commun. 97 (1996) 97.

[43] L. Saviot, D.B. Murray, M. Del Carmen Marco de Lucas, Phys. Rev. B 69 (2004) 113402.

Table 1 Values of densities $\mathrm{q}$, sound velocities $\mathrm{V}_{\mathrm{L}}$ and $\mathrm{V}_{\mathrm{T}}$, elastic constant $\mathrm{C}_{12}$, elastic moduli (L, G, K and E) and Poisson's ratio $\sigma$

\begin{tabular}{llllllllll}
\hline Sample & $\rho\left(\mathrm{g} / \mathrm{cm}^{3}\right)$ & $V_{\mathrm{L}}(\mathrm{m} / \mathrm{s})$ & $V_{\mathrm{T}}(\mathrm{m} / \mathrm{s})$ & $C_{12}(\mathrm{GPa})$ & $L(\mathrm{GPa})$ & $G(\mathrm{GPa})$ & $K(\mathrm{GPa})$ & $E(\mathrm{GPa})$ & $\sigma$ \\
\hline As-prepared & $4.29 \pm 0.01$ & $3310 \pm 8$ & $1847 \pm 9$ & $17.8 \pm 0.7$ & $47.0 \pm 0.3$ & $14.6 \pm 0.2$ & $27.5 \pm 0.5$ & $37.3 \pm 0.4$ & $0.274 \pm 0.007$ \\
Annealed for 5h at $873 \mathrm{~K}$ & $4.57 \pm 0.01$ & $3532 \pm 9$ & $1956 \pm 10$ & $22.0 \pm 0.8$ & $57.0 \pm 0.4$ & $17.5 \pm 0.2$ & $33.7 \pm 0.7$ & $44.7 \pm 0.5$ & $0.278 \pm 0.008$ \\
\hline
\end{tabular}




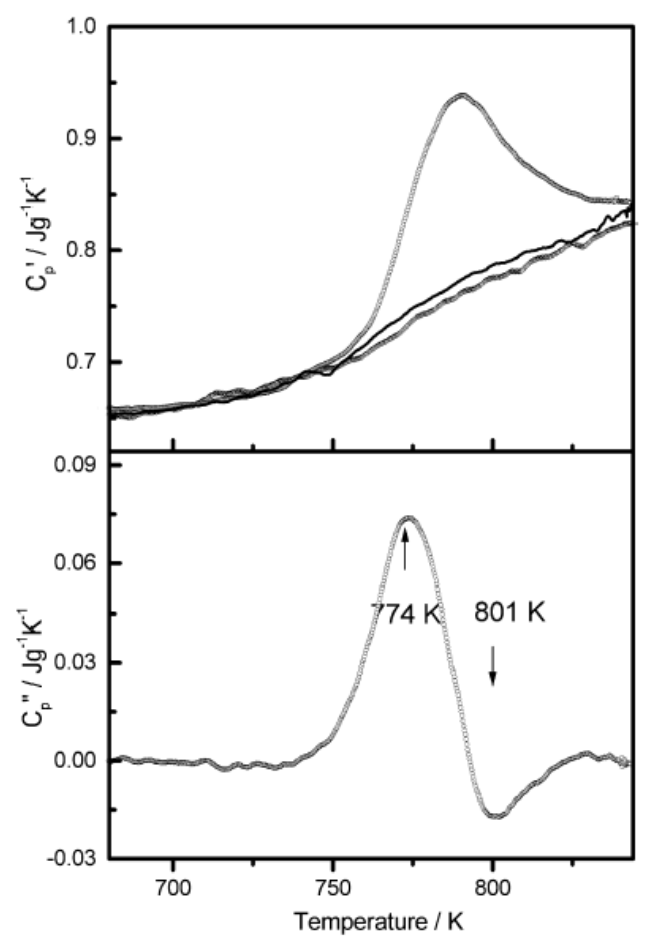

Fig. 1. Temperature dependence of real part of heat capacity for the asprepared CsMWPO glass during heating (open circles) and cooling (diamonds) as well as the CsMWPO glass annealed at $873 \mathrm{~K}$ for $5 \mathrm{~h}$ (line). The lower panel shows temperature dependence of imaginary part of heat capacity for the as-prepared CsMWPO glass.

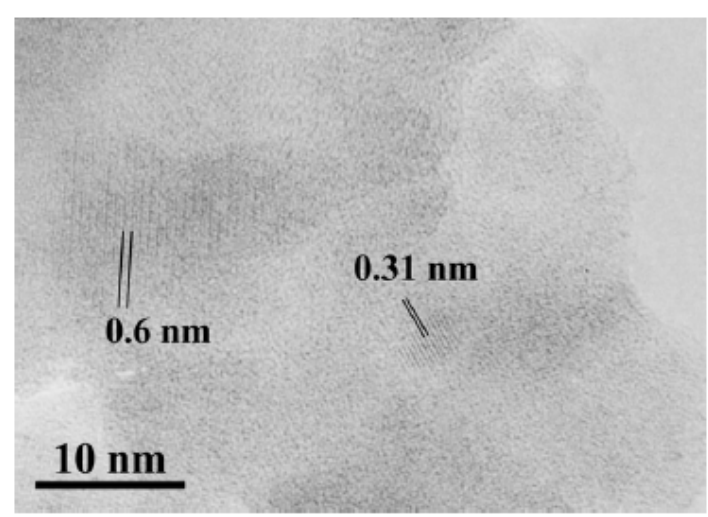

Fig. 2. High-resolution TEM image of the nanocrystallized CsMgWPO glass showing small area with long range order. 


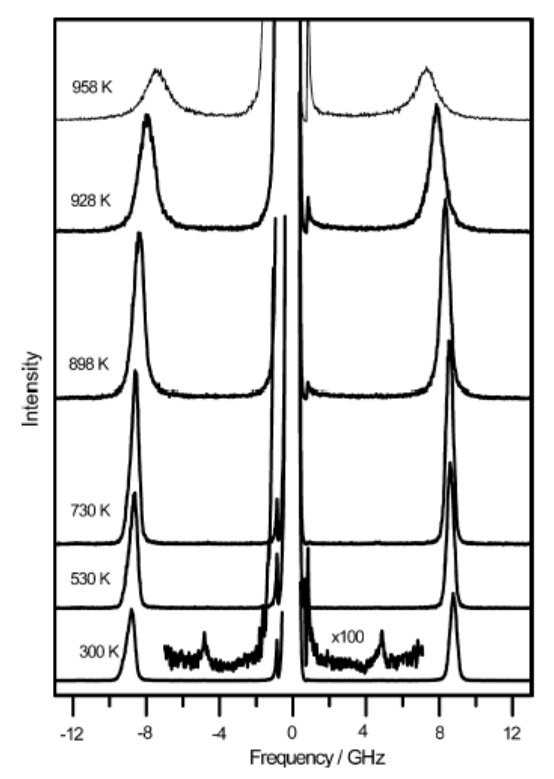

Fig. 3. Representative Brillouin spectra measured at a few temperatures for the CsMWPO glass. Transverse modes are also shown at $300 \mathrm{~K}$ in the plot multiplied by 100 .

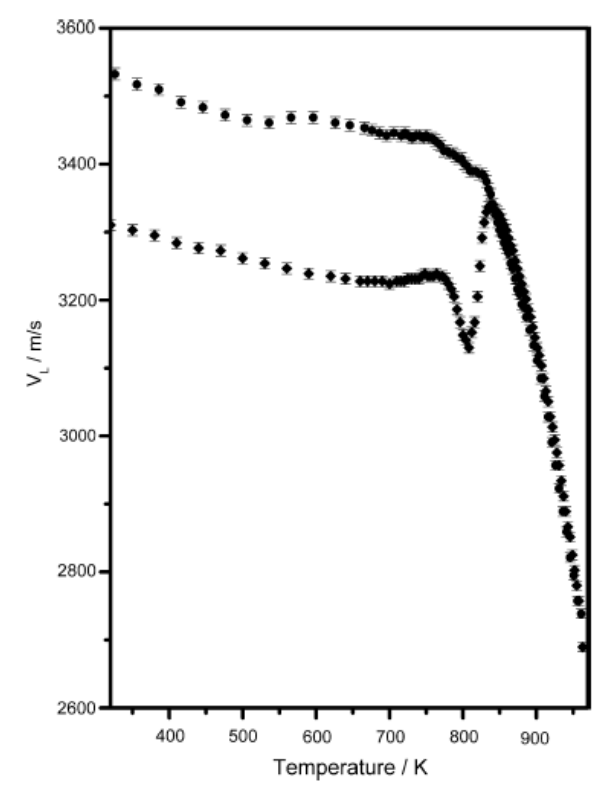

Fig. 4. Temperature dependences of longitudinal sound velocity for the as-prepared glass (diamonds) and the CsMWPO sample annealed at $873 \mathrm{~K}$ for $5 \mathrm{~h}$ (circles). 


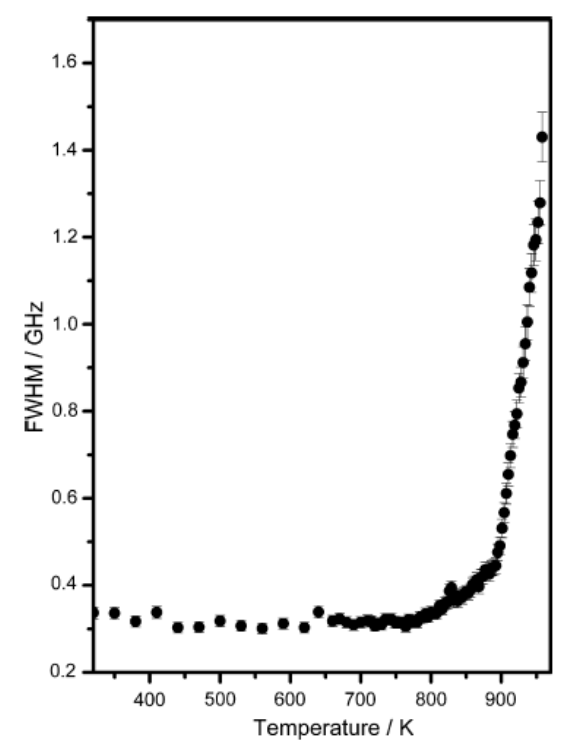

Fig. 5. Temperature dependence of bandwidth of the LA modes for theCsMWPO glass.
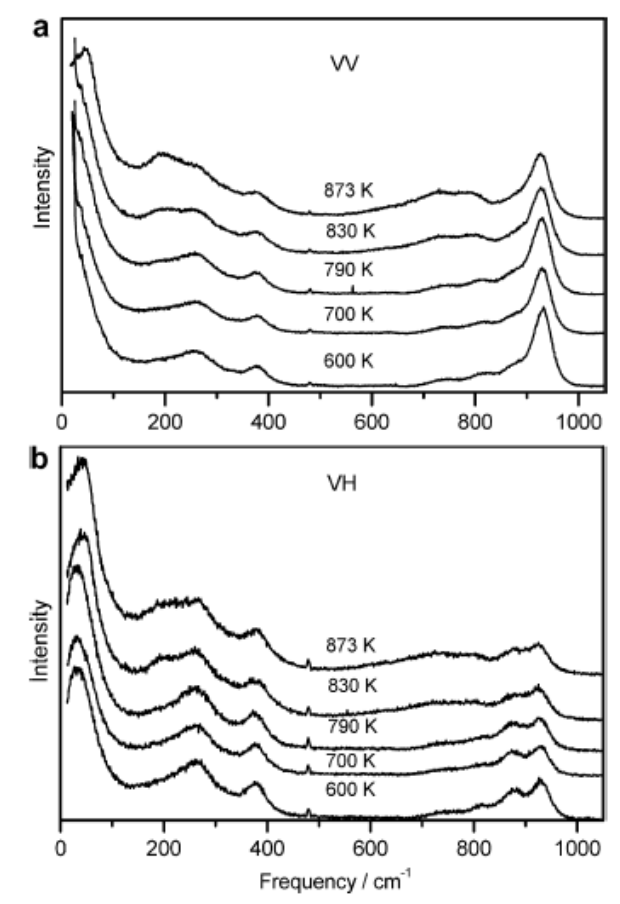

Fig. 6. Raman spectra of the CsMWPO sample at di.erent temperatures in the VV (a) and VH (b) polarization. The spectra were vertically shifted for clarity sake. 


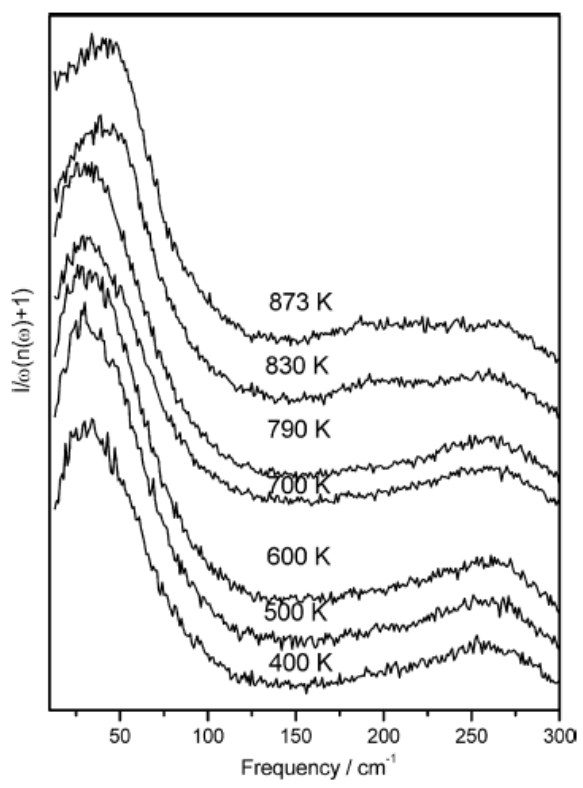

Fig. 7. Low-frequency VH Raman spectra at di.erent temperatures corrected for their frequency and temperature dependence using Eq. (1). The spectra were vertically shifted for clarity sake.
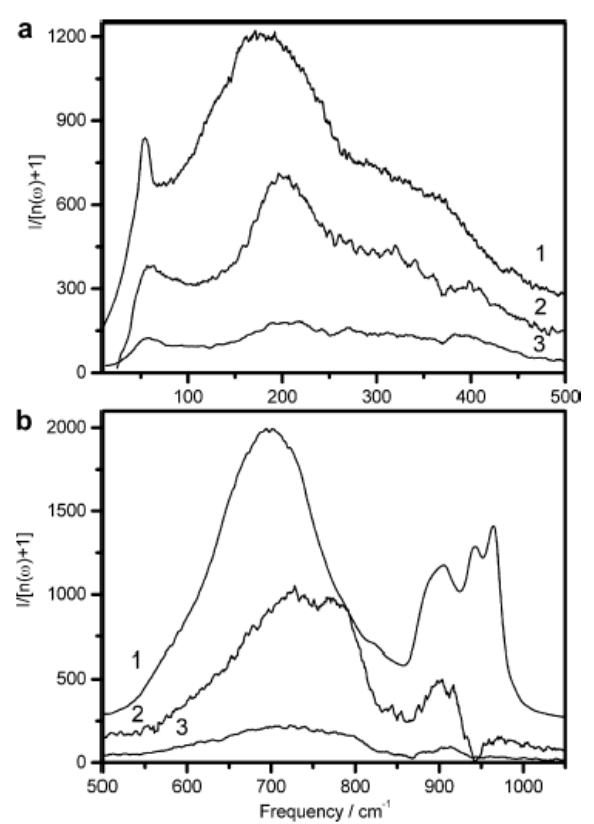

Fig. 8. Low-frequency (a) and high-frequency (b) Raman spectra of $\mathrm{CsMg}_{0.25} \mathrm{~W}_{1.75} \mathrm{O}_{6}$ pyrochlore (plots 1) as well as the VV (plots 2) and VH (plots 3) spectra obtained by subtracting the $793 \mathrm{~K}$ spectra from the $873 \mathrm{~K}$ Raman spectra in Fig. 6. In this procedure the $793 \mathrm{~K}$ spectra were multiplied by factor 0.78 to correct for change in intensity of the amorphous matrix due to partial crystallization. 